\title{
Percentage ${ }^{*},+$ of Adults Aged $\geq 20$ Years Who Consumed Vegetables on a Given Day, by Race and Hispanic Origin ${ }^{\S}$ — United States, 2015-2018
}

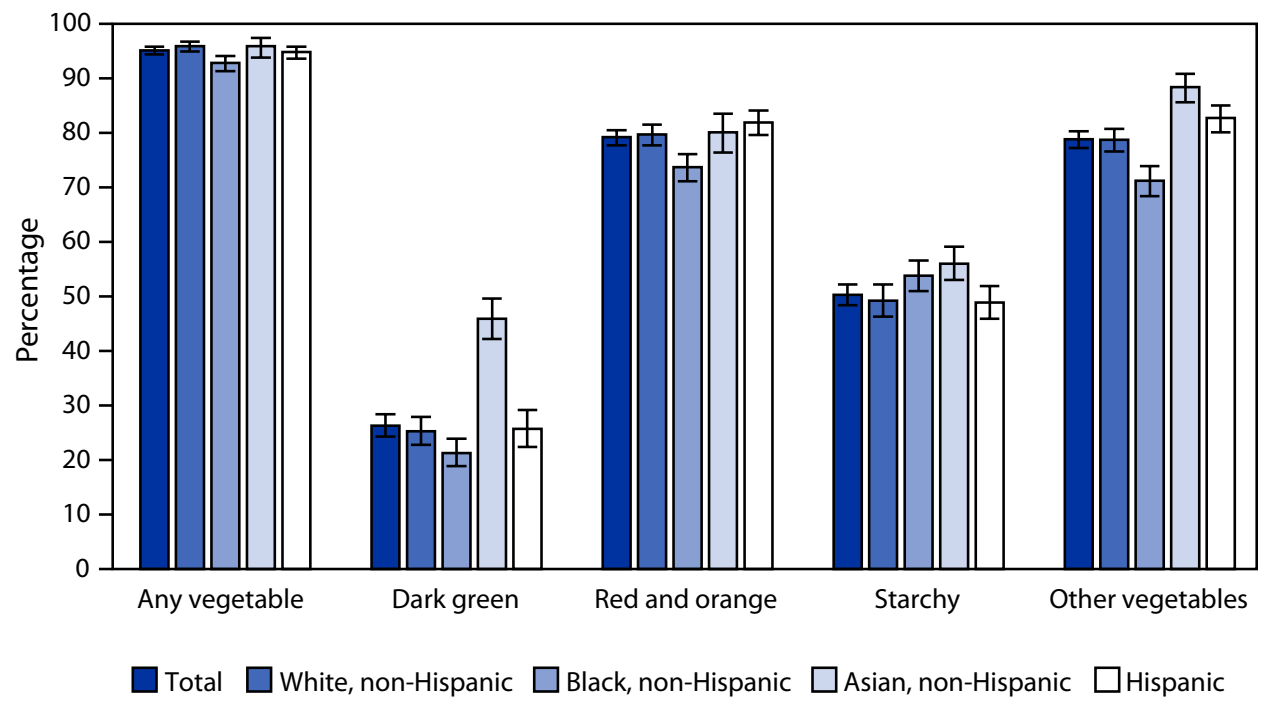

\footnotetext{
* With 95\% confidence intervals indicated with error bars.

† Percentages are based on vegetables reported during the Day 1 24-hour Dietary Recall. Vegetables were defined using the U.S. Department of Agriculture's Food Patterns Equivalents Database food groups (https:// www.ars.usda.gov/ARSUserFiles/80400530/pdf/fped/FPED_1718.pdf), which include dark green vegetables (e.g., spinach, collard greens, and broccoli); red and orange vegetables (e.g., carrots, red peppers, and tomatoes); starchy vegetables (e.g., potatoes, plantains, and cassava); and other vegetables (e.g., cauliflower, string beans, and eggplant).

$\S$ Estimates for persons reporting more than one race are not shown separately but are included in the total.
}

During $2015-2018,95.1 \%$ of adults aged $\geq 20$ years consumed any vegetable, $26.3 \%$ consumed dark green vegetables, $79.2 \%$ consumed red and orange vegetables, $50.3 \%$ consumed starchy vegetables, and $78.8 \%$ consumed other vegetables on a given day. Non-Hispanic Black adults were least likely to consume any vegetable (92.8\%). Non-Hispanic Black adults were also least likely to consume dark green (21.3\%), red and orange (73.7\%), and other vegetables (71.2\%), and non-Hispanic Asian adults were most likely to consume dark green (45.9\%) and other vegetables (88.4\%). Non-Hispanic Black (53.8\%) and non-Hispanic Asian (56.0\%) adults were more likely to consume starchy vegetables.

Sources: Ansai N, Wambogo EA. Fruit and vegetable consumption among adults in the United States, 2015-2018. National Center for Health Statistics (NCHS) data brief, no 397. https://www.cdc.gov/nchs/products/databriefs/db397.htm; NCHS, National Health and Nutrition Examination Survey (NHANES) data, NHANES 2017-2018. https://www.cdc.gov/nchs/nhanes.htm

Reported by: Nicholas Ansai, MPH, qjk0@cdc.gov, 301-458-4385; Edwina Wambogo, PhD; Ana Terry, MS. 